\title{
Diagnosing Cochlear Dead Regions in Children
}

\author{
Alicja N. Malicka, Kevin J. Munro, and Richard J. Baker
}

Objective: A dead region (DR) is defined as a region in the cochlea where inner hair cells and/or neurons are functioning so poorly that a tone producing peak vibration in this region is detected by off-frequency listening, i.e., via a place on the basilar membrane with a characteristic frequency different from that of the tone. The presence of a DR can have a significant effect on the perception of speech. People with and without DRs may differ in the benefit obtained from amplification and require different hearing aid settings. The Threshold Equalizing Noise (TEN) test and psychophysical tuning curves (PTCs) are two procedures used to identify a DR in adults. Because diagnosing a DR involves measuring masked thresholds, and there are reports in the literature that young children perform poorly compared with adults in background noise, it may be possible that the criteria used with adults may not be appropriate when testing children. Therefore, the aim of this study was to evaluate the consistency of the fast-PTC and TEN tests in diagnosing a DR in hearing-impaired children. In addition, the masked thresholds for normal-hearing children were measured with different TEN levels to assess whether any age-related effect in children compared with adults may occur.

Design: Participants were divided into two groups: eight normal-hearing children (16 ears) and 12 hearing-impaired children (21 ears), aged 7 to $13 \mathrm{yr}$. TEN is based on measuring masked threshold in TEN. For normal-hearing participants, the masked thresholds were measured for five levels of noise $(30,40,50,60$, and $70 \mathrm{~dB}$ per averaged equivalent rectangular bandwidth). For hearing-impaired participants, the level of the TEN was selected separately for each ear based on the highest acceptable level minus $5 \mathrm{~dB}$. The TEN test results in hearing-impaired children were further validated by measuring fast-PTCs. The fast-PTC technique involves measuring the level of the narrowband noise masker needed to mask the signal. The center frequency of the masker sweeps across the required frequency range.

Results: The masked thresholds in TEN measured for normal-hearing children were usually below and never higher than $5 \mathrm{~dB}$ above TEN level per averaged equivalent rectangular bandwidth. This suggests that no age-related effect on masked threshold in children compared with adults was observed. All hearing-impaired children were able to perform the TEN test and fast-PTCs. The results of the two tests were consistent in 17 of 21 ears (81\%): eight ears did not show evidence of a DR and nine ears did. In three ears, the criteria for a DR were met on the TEN test, but there was no evidence of a DR on the fast-PTC test. In one ear, the TEN test did not show evidence of DRs at two frequencies, whereas fast-PTCs did.

Conclusions: The results of this study suggest that DRs can be detected in children using the fast-PTC technique and the TEN test interpreted with the adult criteria, which are the most appropriate in terms of specificity and sensitivity. However, in cases in which the masked threshold is 10 to $15 \mathrm{~dB}$ above the TEN level, it is recommended to confirm DR diagnosis with fast-PTC measurement.

(Ear \& Hearing 2010;31;1-•)

\section{INTRODUCTION}

A dead region (DR) is defined as a region in the cochlea where inner hair cells and/or neurons are absent or functioning

School of Psychological Sciences, University of Manchester, Manchester, United Kingdom. so poorly that the tone producing maximum vibration is detected via a different place on the basilar membrane (Moore 2001, 2004). The presence of a DR can have several perceptual consequences: there is evidence of abnormal pitch perception (Huss \& Moore 2005a; Moore \& Carlyon 2005), rapid growth of loudness (McDermott et al. 1998), and distorted perception of pure tones (Florentine \& Houtsma 1983; Huss \& Moore 2005b). An important perceptual consequence, from a clinical perspective, is the effect that the presence of a DR may have on speech perception. Several researchers have investigated the effect of stimulus bandwidth on the ability of people with DRs to understand speech. Some of these studies suggest that providing amplification at frequencies well inside a DR may not provide additional benefit and, in some cases, have a deleterious effect on speech perception (Murray \& Byrne 1986; Vickers et al. 2001; Baer et al. 2002; Vestergaard 2003; Preminger et al. 2005; Vinay \& Moore 2007b; Vinay et al. 2008; Moore \& Vinay 2009). In contrast, Mackersie et al. (2004) showed no difference in performance between participants with and without DRs when the speech material was presented in quiet and in low levels of noise. However, the participants in the study by Mackersie et al. had less severe hearing impairment and less extensive DRs compared with those in some of the previous studies. Therefore, the limited benefit from amplification may be restricted to subjects with extensive DRs. There is no evidence in the literature concerning the effect of a DR on speech perception in children. However, the importance of high-frequency amplification in children with moderate-to-severe hearing impairment has been highlighted in several studies (Stelmachowicz et al. 2001, 2002; Stelmachowicz et al. 2007).

Because the diagnosis and assessment of DRs may be of clinical significance in the fitting of hearing devices and management of hearing impairment (Moore 2004), it is important to evaluate the appropriateness of the adult criteria for diagnosing DRs to the diagnosis of DRs in children.

The extent of a DR may be defined in terms of its edge frequency $\left(f_{\text {edge }}\right)$, which corresponds to the characteristic frequency of the inner hair cell and/or neurons immediately adjacent to the DR (Moore 2001). There are two masking techniques that have been used for diagnosing DRs and establishing $f_{\text {edge }}$ : (1) psychophysical tuning curves (PTCs) and (2) the Threshold Equalizing Noise (TEN) test. A PTC shows the level of masker required to mask a signal fixed in level and frequency as a function of the masker center frequency (Chistovich 1957; Small 1959). The signal is usually a sinusoid, presented at a low sensation level, and the masker is typically a narrowband noise. In normal-hearing listeners, PTCs have a sharp tip at the signal frequency $\left(f_{\text {signal }}\right)$. PTCs are usually broader than normal when cochlear hearing impairment is present; however, the tip still lies close to $f_{\text {signal }}$ (Zwicker \& Schorn 1978; Florentine et al. 1980; Moore 1998; Moore \& Oxenham 1998). In listeners with a DR, the tip of the PTC is shifted away from $f_{\text {signal. }}$. The frequency at the tip of the PTC 
indicates $f_{\text {edge }}$. The traditional PTC measurement procedure is time consuming and unsuitable for clinical use where time is restricted and listeners, such as children, may have limited spans of attention. Sek et al. (2005) developed a method previously used by Zwicker (1974) based on the use of a masker whose frequency sweeps across the required frequency range. However, Sek et al. used narrowband noise instead of a pure tone as a masker. Sek et al. systematically evaluated several parameters crucial for efficient measurement of PTCs such as the rate of change of the masker level, masker bandwidth, and the direction of the masker sweep. They showed that the fast-PTC method produced results that are similar to the traditional PTC measurements in adult listeners. Malicka et al. (2009) used a procedure similar to that of Sek et al. but with normal-hearing children and showed that the fast-PTC method is within the capability of most normalhearing 7- to 10-yr old children. The results of Malicka et al. showed that all children were able to perform the task; however, the tip was defined only in $87 \%$ of the fast-PTCs. Although the variability in tip frequency was higher for children, the mean difference between children and adults was not statistically significant.

The TEN test is based on measuring the masked threshold of a sinusoid in TEN. TEN is based on similar principals as "uniform exciting noise" (developed by Zwicker and Stevens in the early 1950s), i.e., the masked threshold is independent of frequency. The uniform exciting noise produces equal intensity for each critical band, whereas TEN is designed to produce a fixed excitation level as a function of averaged equivalent rectangular bandwidth $\left(\mathrm{ERB}_{\mathrm{N}}\right)$ of the auditory filter at moderate sound levels for young normal-hearing listeners. The original version of the TEN test was designed to measure masked thresholds in dB SPL (Moore et al. 2000), whereas the more recent version $\left(\mathrm{TEN}_{\mathrm{HL}}\right)$ allows measurements to be made in dB HL (Moore et al. 2004). The criteria for diagnosing a DR using the TEN test were evaluated in adults by Moore et al. (2000). Initially, they assessed the masked threshold for various TEN levels with normal-hearing adult listeners. Moore et al. reported that mean masked thresholds were almost equal to TEN level/ERB $\mathrm{E}_{\mathrm{N}}$ with an upper limit of $7 \mathrm{~dB}$ above the TEN level/ERB $\mathrm{E}_{\mathrm{N}}$. The broadened auditory filters in hearing-impaired listeners could increase the masked threshold by a further 2 to $3 \mathrm{~dB}$. Therefore, this resulted in the following diagnostic rule: if the masked threshold in TEN is $10 \mathrm{~dB}$ or more above the TEN level/ERB $\mathrm{E}_{\mathrm{N}}$, and the TEN produces at least $10 \mathrm{~dB}$ of masking, this is indicative of a DR. Moore et al. then validated the TEN test criteria with hearing-impaired adults by comparing TEN test and PTC results (PTCs are assumed to be highly reliable indicators of a DR). They reported good agreement between shifted PTC tips and increase in masked threshold in 17 of 20 ears tested. In the three remaining ears, the TEN test showed false-positive results, i.e., the difference between masked threshold and TEN level/ERB ${ }_{\mathrm{N}}$ was $>10 \mathrm{~dB}$, but the tips of the PTCs were not shifted.

The appropriateness of the TEN criteria for diagnosing a DR in hearing-impaired adults has been confirmed in a number of studies (Huss \& Moore 2003; Kluk \& Moore 2006). In contrast, Summers et al. (2003) attempted to validate the TEN test results by measuring PTCs in hearing-impaired adults and argued that the TEN test does not provide an adequately sensitive and specific indication of a DR. They found only $56 \%$ agreement between the results of the TEN test and the PTC procedure in hearing-impaired listeners when a $10-\mathrm{dB}$ criterion was used (difference between masked threshold and TEN level/ERB $\mathrm{E}_{\mathrm{N}}$ ). However, altering the criterion to $14 \mathrm{~dB}$ changed the level of agreement to $89 \%$. This suggests that, at some frequencies where a DR was not present, the TEN test produced higher masked thresholds than that predicted by Moore et al. (2000). There are additional reports in the literature in which the TEN test produced an abnormally high masking effect, e.g., when testing some hearing-impaired teenagers (Moore et al. 2003) or adults with auditory neuropathy (Vinay \& Moore 2007c).

The TEN test criteria were developed for adult listeners. It is known that psychoacoustic measurements with children are not only more variable than with adults but masked thresholds are also typically higher (Irwin et al. 1986; Hall \& Grose 1990; Veloso et al. 1990; Hall \& Grose 1991; Buss et al. 1999). Therefore, the criteria for the TEN test may not be appropriate for use in the pediatric population. Although the feasibility of using the fast-PTC method has been assessed with normalhearing children, there are no studies reporting the use of fast-PTCs as a tool for diagnosing DRs in hearing-impaired children. Therefore, the aim of this study was to evaluate the consistency of the fast-PTC and $\mathrm{TEN}_{\mathrm{HL}}$ tests in diagnosing DRs in hearing-impaired children. The $\mathrm{TEN}_{\mathrm{HL}}$ test results and criteria were corroborated by measuring fast-PTCs. Previous research with adults suggests that the diagnostic criteria could be adjusted to optimize the validity of the TEN test (Summers et al. 2003); however, it is unknown whether this approach is applicable to school-age children. Therefore, one of the aims of this study was to investigate the sensitivity and specificity of the $\mathrm{TEN}_{\mathrm{HL}}$ test for different diagnostic criteria. In addition, the masked thresholds for normal-hearing children were measured with various TEN levels to assess whether any age-related effect in children compared with adults may occur.

\section{PARTICIPANTS AND METHODS}

\section{Participants}

Eight normal-hearing participants with no history of hearing disorders and absolute thresholds better than $10 \mathrm{~dB}$ HL at 0.5 to $4 \mathrm{kHz}$ were recruited. There were one girl and seven boys, aged between 7 and $12 \mathrm{yr}$. Both ears of each participant were tested. Hearing-impaired participants were selected from three sources: the clinical records from the Audiology Department at the Queens Medical Centre, Nottingham, Manchester Inclusion Service for Children with Hearing Needs, and Mary Hare Grammar School, Newbury. There were 12 experienced hearing aid users (five boys and seven girls, aged between 8 and 13 yr) with high-frequency sensorineural hearing impairment. Both ears of nine participants (18 ears) and one ear from three participants were tested. In two of the latter three cases (5-R and $8-\mathrm{R})$, the hearing impairment in the nontested ear was too severe to conduct the tests; in one case (12-R), the participant completed the tasks for one ear only because of time limitations. Absolute hearing threshold levels, age, and sex of the hearing-impaired participants are shown in Table 1 . The study received ethical approval from the Nottingham Research Ethics Committee 1 (reference number 05/Q2403/146). Written consents were obtained from parents and participating children. Children with a history of developmental disability, based on 
TABLE 1. Age, sex and absolute hearing threshold (dB HL) of the hearing-impaired listeners

\begin{tabular}{|c|c|c|c|c|c|c|c|c|c|c|}
\hline Subject & Sex & Age $(y r)$ & Ear & \multicolumn{7}{|c|}{ Absolute threshold ( $\mathrm{dB} \mathrm{HL})$ at different frequencies $(\mathrm{kHz})$} \\
\hline 1 & M & 10 & L & 35 & 45 & 50 & 60 & 60 & 65 & 65 \\
\hline \multirow[t]{2}{*}{2} & M & 10 & $\mathrm{~L}$ & 40 & 45 & 45 & $80^{*}$ & $80^{*}$ & 65 & 55 \\
\hline & & & $\mathrm{R}$ & 35 & 45 & 60 & 80 & 70 & 60 & 55 \\
\hline 3 & $\mathrm{~F}$ & 8 & L & 50 & 55 & 60 & 60 & 55 & 60 & 65 \\
\hline 4 & & & $\mathrm{R}$ & 20 & 35 & 45 & $85^{\star}$ & $85^{\star}$ & $85^{\star}$ & $85^{\star}$ \\
\hline 5 & $M$ & 11 & L & 70 & 75 & $80^{*}$ & $95^{*}$ & $105^{*}$ & $>110^{*}$ & $>110^{*}$ \\
\hline \multirow[t]{2}{*}{6} & $\mathrm{~F}$ & 9 & L & 45 & 45 & 60 & $75^{*}$ & $65^{\star}$ & 60 & 60 \\
\hline & & & $\mathrm{R}$ & 50 & 50 & 50 & $65^{*}$ & $65^{\star}$ & 60 & 55 \\
\hline \multirow[t]{2}{*}{7} & $M$ & 11 & $\mathrm{~L}$ & 40 & 55 & 60 & 70 & 70 & 70 & 70 \\
\hline & & & $\mathrm{R}$ & 50 & 60 & 65 & 80 & 75 & 75 & 70 \\
\hline 10 & & & $\mathrm{R}$ & 55 & 60 & 65 & 65 & 70 & 70 & 70 \\
\hline \multirow[t]{2}{*}{11} & $\mathrm{~F}$ & 13 & $L$ & 15 & 70 & 75 & 105 & 100 & 95 & 90 \\
\hline & & & R & 30 & $95^{\star}$ & $95^{\star}$ & $110^{*}$ & $110^{*}$ & $110^{*}$ & $110^{*}$ \\
\hline 12 & $\mathrm{~F}$ & 8 & L & 40 & 50 & 60 & 65 & 55 & 30 & 25 \\
\hline
\end{tabular}

${ }^{*}$ Frequencies at which a DR was present (diagnosed using TEN ${ }_{H L}$ test and fast-PTCs).

$D R$, dead region; PTC, psychophysical tuning curve.

medical records and parental report, were excluded from the study.

\section{Equipment and Procedure}

TEN $_{\text {HL }}$ Test $\bullet$ The $\mathrm{TEN}_{\mathrm{HL}}$ compact disc comprises pure tones at $0.5,0.75,1,1.5,2,3$, and $4 \mathrm{kHz}$ and the TEN. Testing at higher frequencies is not required because the presence of a DR at frequencies $\geq 5 \mathrm{kHz}$ is not clinically significant with respect to amplification requirements. According to Vickers et al. (2001) and Baer et al. (2002), it is recommended to amplify up to one octave above $f_{\text {edge }}$. For $f_{\text {edge }}$ of $5 \mathrm{kHz}$, amplification should be provided up to $10 \mathrm{kHz}$. This is consistent with high-frequency amplification recommended for hearing-impaired individuals without any DRs (Stelmachowicz et al. 2001; Vickers et al. 2001; Baer et al. 2002; Stelmachowicz et al. 2002; Stelmachowicz et al. 2007). The stimuli were controlled and amplified using a Kamplex KC35 (Interacoustic, Assens-Denmark) clinical audiometer fitted with 39 telephonics dynamic headphones. The pure tone was fed to one external input channel of the audiometer, and the noise was fed to the other. Calibration was performed before each test session in accordance with Moore et al. (2004). In the $\mathrm{TEN}_{\mathrm{HL}}$ test, the masker level was held constant, and the signal level was adjusted to obtain the masked thresholds (in contrast to the fast-PTC procedure in which the converse was done). The absolute and the masked thresholds in TEN for each participant were measured following the British Society of Audiology recommended procedure (British Society of Audiology 2004), except that smaller audiometric step sizes were used. The initial descending step size was $10 \mathrm{~dB}$. Once the participant stopped responding, thresholds were determined using a 2-dB ascending and 4-dB descending procedure as recommended by Cairns et al. (2007). For normal-hearing participants, the masked thresholds were measured for five levels of noise (30, $40,50,60$, and $\left.70 \mathrm{~dB} / \mathrm{ERB}_{\mathrm{N}}\right)$. For hearing-impaired participants, the level of the TEN was selected separately for each ear based on the highest acceptable level minus $5 \mathrm{~dB}$ (to avoid discomfort during testing) and ranged from 60 to $100 \mathrm{~dB} /$ $E B_{N}$. The ears were initially classified into two groups, with or without DR, according to the adult criteria recommended by Moore et al. as follows: if for specified frequency the masked threshold is $10 \mathrm{~dB}$ or more above TEN level/ERB $\mathrm{N}_{\mathrm{N}}$, and the difference between masked and absolute threshold is $\geq 10 \mathrm{~dB}$, the result indicates presence of a DR at this frequency. In ears in which there was disagreement between the $\mathrm{TEN}_{\mathrm{HL}}$ test and the fast-PTC, the $\mathrm{TEN}_{\mathrm{HL}}$ test was repeated.

Fast-PTC • The software used to run the test was developed by one of the authors (R. J. B.) for use with a Kamplex KC 35 (Interacoustic, Assens-Denmark) clinical audiometer fitted with 39 telephonics dynamic headphones. The attenuation and mixing of the signals was performed using the audiometer under computer control. The PC was additionally equipped with an external 16-bit sound card (Edirol UA-5 Roland DG, Shizuoka-Japan). The masker was generated from low-pass white noise, which was multiplied by a pure tone to obtain a narrowband noise of the appropriate frequency as described by Malicka et al. (2009). The noise bandwidth was $20 \%$ of $f_{\text {signal }}$ for $f_{\text {signal }} \leq 2 \mathrm{kHz}$ and $10 \%$ of $f_{\text {signal }}$ for $f_{\text {signal }}>2 \mathrm{kHz}$. The rate of change of the masker was 0.005 and $0.01 \mathrm{kHz}$ per presentation interval (linear frequency steps) for $f_{\text {signal }}<1 \mathrm{kHz}$ and $f_{\text {signal }} \geq 1 \mathrm{kHz}$, respectively. Fast-PTCs were measured for pulsed pure tones that were sinusoidally amplitude modulated at $8 \mathrm{~Hz}$ to minimize the effect of beat detection, which can affect the shape of the PTC (Kluk \& Moore 2005). The signal duration was $500 \mathrm{msec}$, including $20 \mathrm{msec}$ raised-cosine rise and fall times, and was centered in a $700-\mathrm{msec}$ masker interval 
with the same rise-fall times. There was a 200-msec silent interval between each masker presentation (400 $\mathrm{msec}$ between each signal presentation). The $f_{\text {signal }}$ and masker frequency range were selected on the basis of whether the adult DR criteria were met on the $\mathrm{TEN}_{\mathrm{HL}}$ test. For ears not meeting the criteria, PTCs were measured for two or three frequencies: usually, 1, 2, and $4 \mathrm{kHz}$ and the center frequency of the masker swept from one octave below $f_{\text {signal }}$ up to one octave above $f_{\text {signal. }}$. For ears meeting the criteria for a DR on the $\mathrm{TEN}_{\mathrm{HL}}$ test, PTCs at two $f_{\text {signals }}$ were measured. The first PTC was measured with $f_{\text {signal }}$ one octave below the closest frequency to $f_{\text {edge }}$ for which the $\mathrm{TEN}_{\mathrm{HL}}$ test met the criteria for a DR (border frequency $\left[f_{\text {border }}\right]$ ) and the masker sweep range as above. The second PTC was measured at $f_{\text {border. }}$ In these cases, the masker swept from two octaves below $f_{\text {signal }}$ up to one octave above $f_{\text {signal. }}$ The fast-PTC commenced with the signal presented at 10 $\mathrm{dB}$ sensation level, and the initial masker level was selected according to the severity of the hearing impairment (usually 20 $\mathrm{dB}$ below the signal level). Participants were instructed to press the space bar on the keyboard when the target tone was audible and to release it when the target tone was inaudible. Pressing the space bar increased the masker level in 3-dB step sizes. To provide an objective measure of frequency at the tip $\left(f_{\text {tip }}\right)$, the procedure used by Malicka et al. was used. This involved smoothing the raw data and using a two-point running average of successive reversal points. The frequency at which the minimum point of this line occurred was taken as $f_{\text {tip }}$. In the Results section, only the smoothed line is shown in the figures. Based on the results of Malicka et al. with normalhearing children, the criteria for diagnosing DRs using fast-PTCs were as follows: for an ascending masker sweep direction, the difference between $f_{\text {signal }}$ and $f_{\text {tip }}$ of $>25 \%$ of $f_{\text {signal }}$ toward higher frequencies or $20 \%$ toward lower frequencies was taken as an indication of a DR.

\section{RESULTS}

\section{Normal-Hearing Children}

The results of the $\mathrm{TEN}_{\mathrm{HL}}$ test for the normal-hearing children are presented in Figure 1. The mean masked thresholds measured with TEN at 30, 40, and $50 \mathrm{~dB} / \mathrm{ERB}_{\mathrm{N}}$ were almost invariant across all frequencies and below the TEN level/ERB ${ }_{\mathrm{N}}$, regardless of $f_{\text {signal }}$. For TEN levels of 60 and 70 $\mathrm{dB} / \mathrm{ERB}_{\mathrm{N}}$, the relative increase in masked threshold was greater at high frequencies. However, individual masked thresholds measured in normal-hearing children were never increased $>5 \mathrm{~dB}$ above the TEN level/ERB $\mathrm{ER}_{\mathrm{N}}$, regardless of noise level and test frequency. The difference between masked threshold and TEN level/ERB $\mathrm{E}_{\mathrm{N}}$ is crucial because it defines the criterion for diagnosing a DR. The results of this study with normal-hearing children are comparable with those reported previously with normal-hearing adults (Moore et al. 2000) and teenagers (Moore et al. 2003). Therefore, it is suggestive that the adult $10-\mathrm{dB}$ criterion may be appropriate for diagnosing DRs in hearing-impaired children. On the other hand, because of limited listening experience, hearing-impaired children may be more prone to masking, and the criteria developed for normal-hearing participants may need to be altered for hearingimpaired individuals.

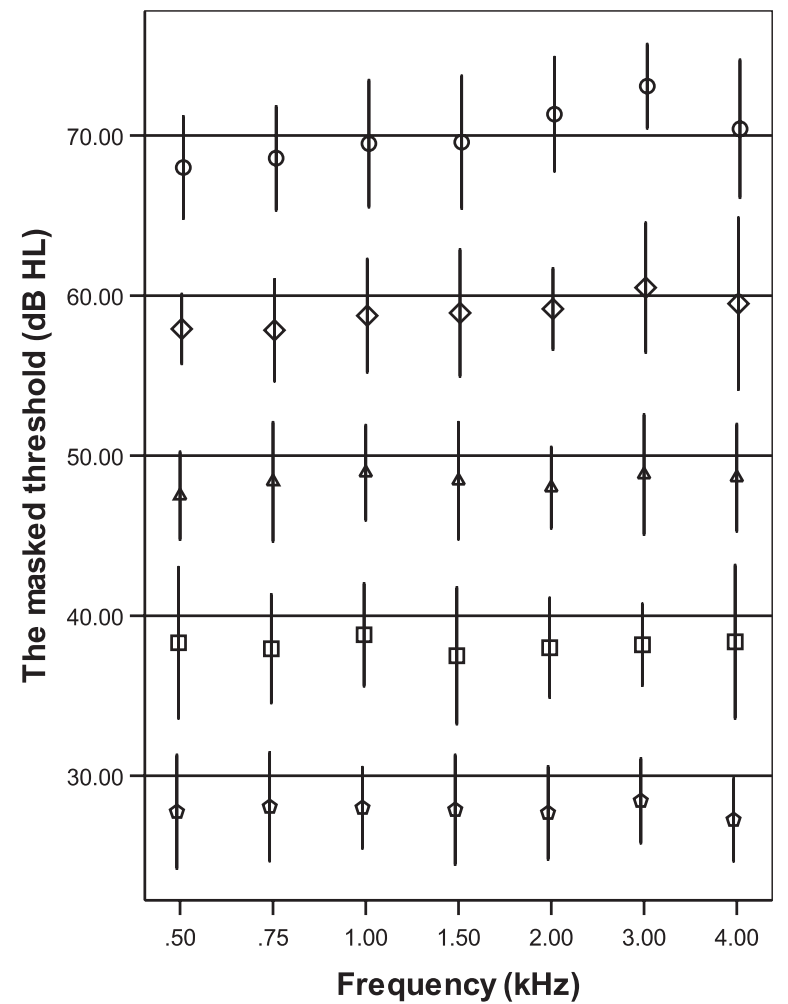

Fig. 1. TEN $\mathrm{HL}_{\mathrm{HL}}$ results for 16 ears of normal-hearing children. The symbols represent the mean masked thresholds measured for different TEN levels/ $E R B_{N}$, and the error bars show $\pm 2 \mathrm{SD}$. TEN, Threshold Equalizing Noise; $E R B_{N}$, averaged equivalent rectangular bandwidth.

\section{Hearing-Impaired Children}

All children were able to perform the $\mathrm{TEN}_{\mathrm{HL}}$ test and the fast-PTC test. Both procedures were used to establish the presence or absence of DRs in 21 ears. Figure 2 shows the results for the eight ears that did not show evidence of a DR on the $\mathrm{TEN}_{\mathrm{HL}}$ test and the fast-PTC test. Each column represents findings for one ear. The top row shows $\mathrm{TEN}_{\mathrm{HL}}$ test results obtained with the TEN level specified in the bottom left corner of each panel. The absolute and masked thresholds are represented by filled and open squares, respectively. The masked thresholds for all tested frequencies were within $10 \mathrm{~dB}$ (mean $3.6 \mathrm{~dB}$, range 0 to $9 \mathrm{~dB}$ ) of the TEN level/ERB $\mathrm{N}_{\mathrm{N}}$. The bottom row shows fast-PTCs (smoothed lines). The fast-PTCs presented in Figure 2 were measured at 1, 2, and $4 \mathrm{kHz}$ in all ears, except 2-R in which the PTCs were measured at 0.5 and $1 \mathrm{kHz}$ and $11-\mathrm{L}$ in which the PTCs were measured at 2 and $3 \mathrm{kHz}$ only. The open symbols represent $f_{\text {tip }}$ of the PTC. The filled symbols (with respective shape) represent the $f_{\text {signal }}$ for which the PTC was measured. Ideally, for participants without any DR, the open and the filled symbols should indicate the same frequency. However, in practice, the $f_{\text {tip }}$ is often shifted away from $f_{\text {signal }}$, and the magnitude of this shift is considered as a criterion for diagnosing a DR. The mean shift in the tip of PTCs presented in Figure 2 was $8.7 \%$ of $f_{\text {signal }}$ (range 0.3 to $18 \%$, which is consistent with the $\mathrm{TEN}_{\mathrm{HL}}$ test results and the absence of a DR in these ears.

Figure 3 shows results for nine ears that met the criteria for a DR both on the TEN $\mathrm{HL}_{\mathrm{HL}}$ test and on the fast-PTC test. Each column shows findings for one ear. The $\mathrm{TEN}_{\mathrm{HL}}$ test results are 
TEN test: $\square$-absolute threshold, $\square$-masked threshold measured with specified TEN level

Fast-PTC: Fsignal $\bullet-0.5 \mathrm{kHz} \star-1 \mathrm{kHz} \bullet-2 \mathrm{kHz} \bullet-3 \mathrm{kHz} \nabla-4 \mathrm{kHz}$

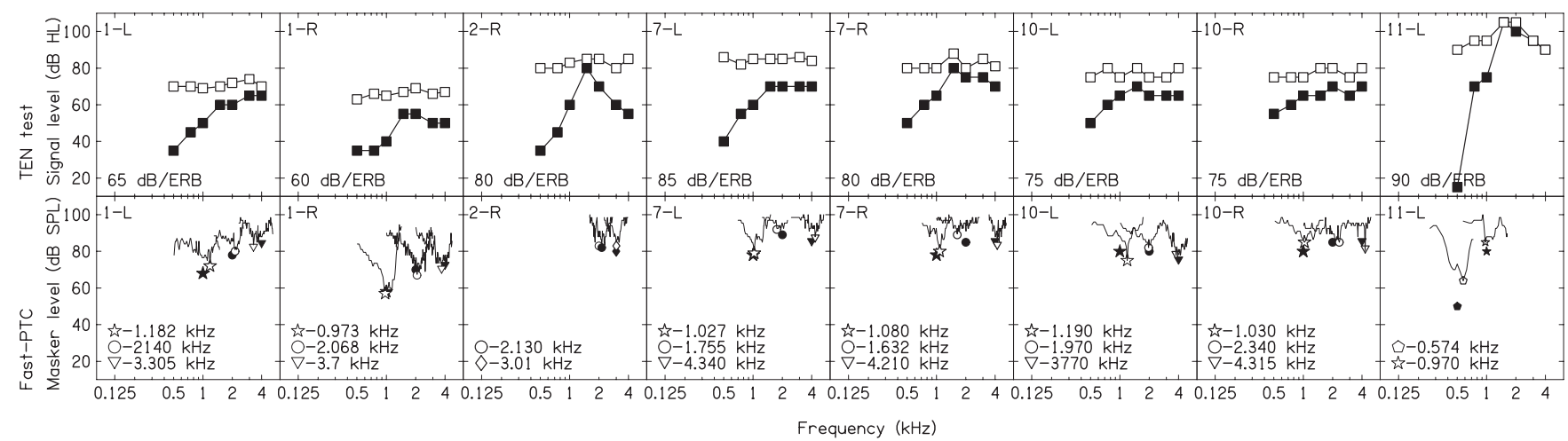

Fig. 2. Results for the eight ears without any evidence of a DR on the TEN $\mathrm{HL}_{\mathrm{HL}}$ test and the fast-PTC test. Each column contains results for one ear. The TEN $\mathrm{HL}_{\mathrm{L}}$ test (top row) was measured with the TEN level specified in the bottom left corner of each panel. Absolute threshold and masked threshold are represented by filled and open squares, respectively. The PTC results are shown in the bottom row. The smoothed line represents two-point running average of successive reversal points. The open symbols correspond to $f_{\text {tip }}$ of the PTCs measured for $f_{\text {signal }}$ represented by filled symbol with respective shape. The value of $f_{\text {tip }}$ for each PTC is given in the left bottom corner of each panel containing PTCs. DR, dead region; PTC, psychophysical tuning curve; TEN, Threshold Equalizing Noise; $f_{\text {tip }}$ tip frequency; $f_{\text {signal, }}$ signal frequency.

shown in the first row, followed by fast-PTCs measured at $f_{\text {signal }}$ outside the DR (middle row) and finally PTCs measured at $f_{\text {border }}$ (bottom row). The $\mathrm{TEN}_{\mathrm{HL}}$ results are presented in the same format as Figure 1. The frequencies for which the criteria for a DR were met on the $\mathrm{TEN}_{\mathrm{HL}}$ test are represented by single hashed area on the figures.

The mean masked threshold measured for frequencies outside the DR was $5.5 \mathrm{~dB}$ (range 0 to $9 \mathrm{~dB}$ ) above TEN level/ERB $\mathrm{E}_{\mathrm{N}}$. For frequencies falling within a DR, the masked threshold was increased $\geq 15 \mathrm{~dB}$ for all ears, except 9-L and 9-R, at 0.75 and $1 \mathrm{kHz}$, in which the masked threshold was 10 $\mathrm{dB}$ above the TEN level/ERB $\mathrm{E}_{\mathrm{N}}$. The mean shift in the tip of the
PTCs obtained with $f_{\text {signal }}$ outside the DR was $7.5 \%$ of $f_{\text {signal }}$ (range 0.7 to $20 \%$ ). The bottom row shows PTCs measured with $f_{\text {signal }}$ equal to $f_{\text {border. }}$. For these PTCs, the shift varied between 33.1 and $65 \%$ of $f_{\text {signal }}$. Six ears with severe to profound degrees of hearing impairment showed extensive DRs limited only from the low-frequency side. In all these ears, the fast-PTC measured at $f_{\text {border }}$ displayed shifted tips consistent with the presence of a DR. The audiometer output level was not high enough to establish the masked thresholds at some frequencies (4-L at 2 and $4 \mathrm{kHz} ; 8-\mathrm{L}$ at $1 \mathrm{kHz} ; 9-\mathrm{L}$ at $1.5,2$, and $3 \mathrm{kHz}$; and $11-\mathrm{R}$ above $0.75 \mathrm{kHz}$ ), but the masked threshold was at least $20 \mathrm{~dB}$ above TEN level/ERB $\mathrm{E}_{\mathrm{N}}$ and produced at

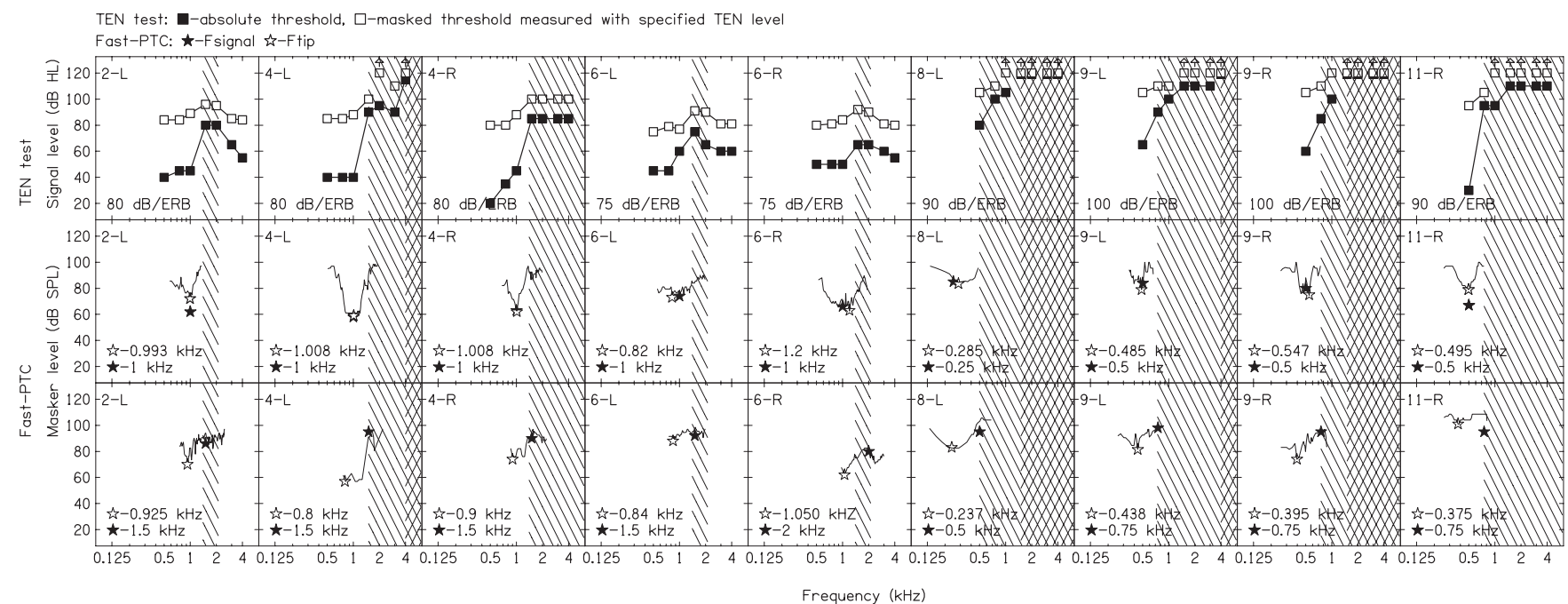

Fig. 3. Results for the nine ears with evidence of DRs on the TEN $\mathrm{HL}_{\mathrm{L}}$ test and the fast-PTC test. Each column contains results for one ear. Top row shows results of the TEN $\mathrm{HL}_{\mathrm{H}}$ test as shown in Fig. 2. The symbols with upward-pointing arrows indicate cases in which the threshold was too high to be measured. Single hashed area indicates frequencies where DR criteria are met on the TEN $\mathrm{HL}_{\mathrm{HL}}$ test, i.e., the difference between masked threshold and TEN level/ERB is $\geq 10 \mathrm{~dB}$, and the difference between masked and absolute threshold is $\geq 10 \mathrm{~dB}$. The double hashed area indicates frequencies where the output level of the audiometer was not sufficient to measure absolute and/or masked threshold, but the presence of DR at these frequencies is likely. The middle row shows PTCs measured with $f_{\text {signal }}$ outside a DR. Bottom row shows PTCs measured with $f_{\text {signal }}$ from inside the DR. The $f_{\text {tip }}$ (open star) and $f_{\text {signal }}$ (filled star) values are given in the left corner of each panel containing PTC. DR, dead region; PTC, psychophysical tuning curve; TEN, Threshold Equalizing Noise; ERB, equivalent rectangular bandwidth; $f_{\text {signal, }}$ signal frequency; $f_{\text {tip }}$ tip frequency. 
Fig. 4. The findings for four ears showing disagreement between the TEN ${ }_{\mathrm{HL}}$ test and fast-PTCs. Same format as Fig. 3. PTC, psychophysical tuning curve.

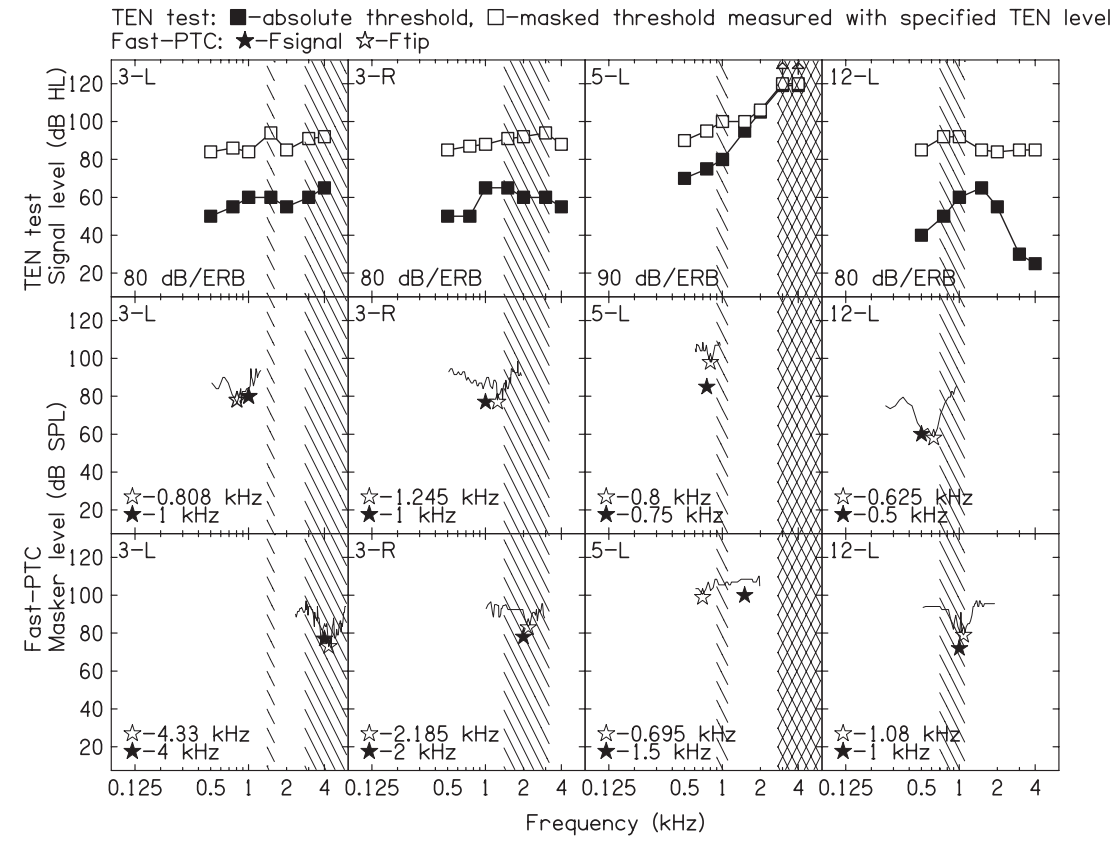

least $10 \mathrm{~dB}$ of masking. Therefore, despite the limitations of the audiometer, the presence of a DR was established by the $\mathrm{TEN}_{\mathrm{HL}}$ test at these frequencies.

The interpretation of the $\mathrm{TEN}_{\mathrm{HL}}$ test becomes more complicated at frequencies where it was not possible to establish a relationship between masked and absolute threshold because of severity of hearing impairment (8-L above $1 \mathrm{kHz}$; 9L at $4 \mathrm{kHz}$; and 9-R above $1 \mathrm{kHz}$ ). In these cases, the results of $\mathrm{TEN}_{\mathrm{HL}}$ test are inconclusive, although it is likely that a DR is present based on the severity of hearing impairment (double hashed area on the graph). For one ear (8-L), it was not possible to determine $f_{\text {edge }}$ based on the TEN test results. However, the PTC measured with an $f_{\text {signal }}$ of $0.5 \mathrm{kHz}$ showed evidence of a DR because the tip was shifted toward low frequencies at 0.237 $\mathrm{kHz}\left(52.6 \%\right.$ of $\left.f_{\text {signal }}\right)$. The shift in the tip of the PTC measured with an $f_{\text {signal }}$ of $0.25 \mathrm{kHz}$ was within $14 \%$ of $f_{\text {signal }}$, and this is consistent with no DR at this frequency.

There were three examples of "island DRs," i.e., limited from low- and high-frequency side. According to the $\mathrm{TEN}_{\mathrm{HL}}$ test for ears $2-\mathrm{L}, 6-\mathrm{L}$, and 6-R, the low-frequency $f_{\text {edge }}$ was located between 1 and $1.5 \mathrm{kHz}$, and the high-frequency $f_{\text {edge }}$ was located between 2 and $3 \mathrm{kHz}$. For these ears, $f_{\text {tip }}$ of the PTCs, measured with an $f_{\text {signal }}$ of $1 \mathrm{kHz}$ (outside DR) was within $20 \%$ of $f_{\text {signal }}$. The PTCs measured at $f_{\text {border }}$ : for ear $2-\mathrm{L}$ and 6-L at $1.5 \mathrm{kHz}$ (low-frequency $f_{\text {border }}$ ) and for $6-\mathrm{R}$ at $2 \mathrm{kHz}$ (high-frequency $f_{\text {border }}$ ) displayed tips shifted toward $1 \mathrm{kHz}$ irrespective of $f_{\text {signal }}$. The magnitude of the shift varied between 38.3 and $47.5 \%$ of $f_{\text {signal }}$. Therefore, it may be assumed that these signals are detected via a filter with characteristic frequency close to $1 \mathrm{kHz}$. Interestingly, for 6-R, the other tip is visible between 2 and $3 \mathrm{kHz}$, which may suggest that both places are responsible for detection of $2-\mathrm{kHz}$ signal.

Four of the 21 ears showed a disagreement between the outcome of the $\mathrm{TEN}_{\mathrm{HL}}$ test and the fast-PTC test (3-L, 3-R, $5-\mathrm{L}$, and 12-L). The results for these ears are presented in Figure 4. In these ears, the $\mathrm{TEN}_{\mathrm{HL}}$ test was conducted on two occasions. The mean test-retest difference was $1.6 \mathrm{~dB}(\mathrm{SD}=$
1.7), and there was no change in diagnostic category; therefore, only data from the first attempt were analyzed and shown on the graphs. For 3-L, the mean masked threshold was $8 \mathrm{~dB}$ above the TEN level (range 4 to $14 \mathrm{~dB}$ ) showing abnormally high masking for $1.5,3$, and $4 \mathrm{kHz}$. The $f_{\text {tip }}$ of the PTC measured with an $f_{\text {signal }}$ of $1 \mathrm{kHz}$ (no DR on the TEN $\mathrm{HL}_{\mathrm{HL}}$ test) was within $19.2 \%$ of $f_{\text {signal }}$, although shifted toward lower frequencies (opposite to masker sweep direction) but still in line with no DR at this frequency. The $f_{\text {tip }}$ of the PTC measured with an $f_{\text {signal }}$ of $4 \mathrm{kHz}$ (the criteria for a DR met on the $\mathrm{TEN}_{\mathrm{HL}}$ test) was within $8.3 \%$ of $f_{\text {signal }}$. Therefore, the results of TEN $\mathrm{TL}_{\mathrm{HL}}$ and fast-PTCs are in agreement at $1 \mathrm{kHz}$ but not at $4 \mathrm{kHz}$. A similar pattern is present for ear 3-R. The mean masked threshold was $9.3 \mathrm{~dB}$ (range 5 to $14 \mathrm{~dB}$ ) above the TEN level, and the criteria for a DR were met at $1.5,2$, and $3 \mathrm{kHz}$. The PTCs measured for 1 and $2 \mathrm{kHz}$ did not show evidence of DR (shift in the tip, 24.5 and $9.3 \%$ of $f_{\text {signal }}$, respectively). Consequently, the results of both tests at $1 \mathrm{kHz}$ are consistent with no $\mathrm{DR}$, whereas at $2 \mathrm{kHz}$, the $\mathrm{TEN}_{\mathrm{HL}}$ test results remain in disagreement because there is no evidence of off-frequency listening on the fast-PTC test. For ear 12-L, the mean masked threshold was $6.9 \mathrm{~dB}$ (range 4 to $12 \mathrm{~dB}$ ) above the TEN level. The criteria for DR were met on $\mathrm{TEN}_{\mathrm{HL}}$ at 0.75 and $1 \mathrm{kHz}$ (masked threshold $12 \mathrm{~dB}$ above the TEN level). The fast-PTCs measured at $0.5 \mathrm{kHz}$ (outside the DR according to the TEN $\mathrm{HL}_{\mathrm{HL}}$ test) displayed a tip shifted toward higher frequencies. The magnitude of the shift was $25 \%$ of $f_{\text {signal }}$, which is borderline abnormal using the criteria specified above. At $1 \mathrm{kHz}$, the fast-PTC showed a tip within $8 \%$ of $f_{\text {signal }}$, which suggests no $\mathrm{DR}$, and this remains in contrast to the $\mathrm{TEN}_{\mathrm{HL}}$ test results.

A different pattern of results was observed for ear 5-L. The $\mathrm{TEN}_{\mathrm{HL}}$ test indicated $f_{\text {border }}$ at $1 \mathrm{kHz}$ because the masked threshold was $10 \mathrm{~dB}$ above TEN level and $20 \mathrm{~dB}$ above the absolute threshold. It would be expected that if the TEN level was high enough to produce masking at $f_{\text {border }}$, the difference between masked and absolute threshold should be carried forward toward higher frequencies (where the hearing impair- 
ment is more severe). However, it was not the case at 1.5 and $2 \mathrm{kHz}$, where the masked thresholds were 10 and $16 \mathrm{~dB}$ above TEN level, respectively, but $<10 \mathrm{~dB}$ above the absolute threshold. The $f_{\text {tip }}$ of the fast-PTCs measured with an $f_{\text {signal }}$ of 0.75 and $1.5 \mathrm{kHz}$ was $0.8 \mathrm{kHz}$ (within $6.7 \%$ of $f_{\text {signal }}$ ) and 0.695 $\mathrm{kHz}$ (within $53.7 \%$ of $f_{\text {signal }}$ ), respectively, and this confirms $f_{\text {edge }}$ around $0.75 \mathrm{kHz}$ and presence of a DR at $1.5 \mathrm{kHz}$.

\section{DISCUSSION}

The $\mathrm{TEN}_{\mathrm{HL}}$ test and the fast-PTC procedure were examined for use with school-age children using equipment commonly available in the clinic. Both techniques are within the capability of children of this age because all children were able to complete the tasks. Thus, it should be expected that the results of both tests will give the same classification for the presence or absence of a DR. In this study, the $\mathrm{TEN}_{\mathrm{HL}}$ test results interpreted using the adult $10-\mathrm{dB}$ criteria were verified by measuring fast-PTCs as the standard reference. In 17 ears $(81 \%)$, results obtained using both techniques were in agreement: nine ears did not meet the criteria for a DR and eight met the criteria. In four ears $(19 \%)$, the results were in disagreement. This agreement rate is consistent with previous studies using adult listeners (Moore et al. 2000; Kluk \& Moore 2006) but in contrast with the findings of Summers et al. (2003). The discrepancy in the outcome of the TEN tests and the fast-PTC tests reported previously was thought to be due to false-positive results on the TEN test. The 56\% agreement rate reported by Summers et al. increased to $89 \%$ after changing the $\mathrm{TEN}_{\mathrm{HL}}$ test criterion referring to a difference between the masked threshold and the TEN level/ERB ${ }_{\mathrm{N}}$ to $14 \mathrm{~dB}$.

On the basis of the findings of this study, the results of the $\mathrm{TEN}_{\mathrm{HL}}$ test and the PTC procedure were in agreement when the masked threshold in TEN was $<10 \mathrm{~dB}$ (no DR) or $\geq 15 \mathrm{~dB}$

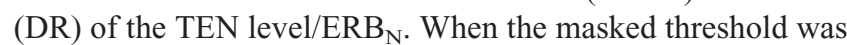
increased 11 to $14 \mathrm{~dB}$ above the TEN level/ERB $\mathrm{N}_{\mathrm{N}}$ the disagreement in the outcomes of both tests was apparent (3-L, 3-R, and 12-L). The fast-PTCs have been successfully used as a technique for identifying off-frequency listening in adults (Summers et al. 2003; Sek et al. 2005; Kluk \& Moore 2006) and can be reliably used with school-age children (Malicka et al. 2009). Therefore, it was assumed that the cases of disagreement are most likely due to false results on the $\mathrm{TEN}_{\mathrm{HL}}$ test. False positives on the $\mathrm{TEN}_{\mathrm{HL}}$ test have also been reported in previous studies with adults (Moore et al. 2000; Summers et al. 2003). One of the approaches to overcome the disagreement is to alter the $\mathrm{TEN}_{\mathrm{HL}}$ test diagnostic criterion as done by Summers et al. (2003). However, adjusting the difference between masked threshold and TEN level/ERB ${ }_{\mathrm{N}}$ indicating the presence of a DR from 10 to $14 \mathrm{~dB}$ did not increase the agreement rate because it reduced the false positives but increased false-negative results. Table 2 shows how a change in $\mathrm{TEN}_{\mathrm{HL}}$ test criterion affects sensitivity and specificity of the test. Sensitivity and specificity (combined) shows the highest value when the $\mathrm{TEN}_{\mathrm{HL}}$ test results were interpreted using the $10-\mathrm{dB}$ criterion. Therefore, on the basis of the results of this study, the adult criteria proposed by Moore et al. (2000) seem to be the most appropriate when diagnosing a DR in children. However, in one case (5-L), the findings cannot be explained according to the theory of off-frequency listening because of the presence of a DR. The masked thresholds at two frequen-
TABLE 2. Sensitivity and specificity for different $T E N_{H L}$ diagnostic criteria (difference between masked threshold and TEN level/ERB N $_{\text {) }}$

\begin{tabular}{ccc}
\hline TEN $_{\mathrm{HL}}$ criterion $(\mathrm{dB})$ & Sensitivity & Specificity \\
\hline$\geq 10$ & $10 / 10(100 \%)$ & $34 / 37(92 \%)$ \\
$\geq 11$ & $7 / 10(70 \%)$ & $34 / 37(92 \%)$ \\
$\geq 13$ & $7 / 10(70 \%)$ & $37 / 37(100 \%)$
\end{tabular}

Sensitivity is defined as number of frequencies with evidence of a $D R$ on the TEN $N_{H L}$ test divided by number of frequencies with evidence of a DR on PTCs. Specificity is defined as number of frequencies without evidence of a DR on the TEN ${ }_{H L}$ test divided by number frequencies with no evidence of a DR on PTCs.

TEN, Threshold Equalizing Noise; $E R B_{N}$, averaged equivalent rectangular bandwidth; DR, dead region; PTC, psychophysical tuning curve.

cies inside the DR were not increased above absolute threshold as expected because the masker was sufficient enough to produce masking at $f_{\text {border }}$. One of the explanations for this phenomenon could be that the listener is attending to distortion products instead of components at the target tone frequency, which could potentially occur due to high stimulus presentation levels. However, further analysis of the stimulus spectrum for this measurement condition using an artificial ear (B\&K type 4152) and spectrum analyzer (B\&K type 2250) did not show the presence of any additional spectral components available for detection to the listener. Therefore, the $\mathrm{TEN}_{\mathrm{HL}}$ results for $5-\mathrm{L}$ at 1.5 and $2 \mathrm{kHz}$ remain unexplained.

It has been suggested that the fast-PTC procedure provides more precise estimation of $f_{\text {edge }}$ of DR than the TEN $\mathrm{HL}_{\mathrm{HL}}$ test in adult listeners (Moore 2004; Sek et al. 2005; Kluk \& Moore 2006). However, the large and variable shift in $f_{\text {tip }}$ observed in normal-hearing (Malicka et al. 2009) and hearing-impaired children (this study) suggests that the estimation of $f_{\text {edge }}$ is not more precise than that identified by the $\mathrm{TEN}_{\mathrm{HL}}$ test. Furthermore, the time required to complete the $\mathrm{TEN}_{\mathrm{HL}}$ test (about 15 min per ear) is shorter than the time required to complete the fast-PTCs (seven frequencies $\times$ approximately 5 min each fast-PTC measurement per ear). However, the fast-PTCs are considered a more reliable tool for diagnosing DRs. Therefore, in clinical practice, it would be recommended to use the fast-PTC procedure to confirm the $\mathrm{TEN}_{\mathrm{HL}}$ test results (especially at frequencies where the masked threshold is 10 to $14 \mathrm{~dB}$ above the TEN level/ERB $\mathrm{N}_{\mathrm{N}}$ ) rather than a separate tool for more precise estimation of $f_{\text {edge }}$.

Interestingly, participants who produced conflicting results on the $\mathrm{TEN}_{\mathrm{HL}}$ test also experienced more difficulties when completing the fast-PTC test. This resulted in relatively large shifts in the tips of PTCs measured with $f_{\text {signal }}$ outside DR (as indicated by $\mathrm{TEN}_{\mathrm{HL}}$ test). Furthermore, the mean masked threshold in TEN across all tested frequencies for those ears (8.1 dB) was considerably higher than that for ears without a DR (3.6 dB) and for ears with DRs at frequencies outside a DR $(5.5 \mathrm{~dB})$. One of the explanations for these findings may be that problems in central auditory processing are responsible, at least in part, for the difficulties in attending to and extracting signals from the background noise. The mean test-retest repeatability on $\mathrm{TEN}_{\mathrm{HL}}$ test in these participants was good, and no change of diagnostic category was observed on retest. However, without test-retest data for both measures in hearing-impaired children, the reason for this finding remains unclear.

Generally, the mean masked thresholds measured in the hearing-impaired children were higher than those previously 
reported with hearing-impaired adults (Moore et al. 2000; Moore et al. 2004; Kluk \& Moore 2006; Vinay \& Moore 2007a) and hearing-impaired teenagers (Moore et al. 2003). There are also reports in the literature that young children perform poorly in background noise compared with adults (Irwin et al. 1986; Allen et al. 1989; Allen \& Wightman 1992, 1994, 1995) but usually reach adult-like performance by the age of 10 to $12 \mathrm{yr}$ (Schneider et al. 1989; Werner \& Marean 1996). To assess whether or not the age of children affects the ability to detect a signal in TEN, a group of normal-hearing children was tested. The masked thresholds measured for TEN of low intensity $\left(30,40\right.$, and $\left.50 \mathrm{~dB} / \mathrm{ERB}_{\mathrm{N}}\right)$ were below the TEN level/ERB $B_{N}$ irrespective of tested frequency. For noise levels of 60 and $70 \mathrm{~dB} / \mathrm{ERB}_{\mathrm{N}}$, the masked thresholds increase relative to the TEN level/ERB ${ }_{\mathrm{N}}$, especially at high frequencies; however, they were never $>5 \mathrm{~dB}$ above TEN level/ERB $\mathrm{E}_{\mathrm{N}}$. The greater masking effect for higher levels at high frequencies may be explained in terms of the broader tuning at high levels related to an effect of spread of excitation along the basilar membrane (Glasberg \& Moore 1990; Rosen \& Baker 1994; Ruggero et al. 1997; Baker \& Rosen 2006). The results of this study are in line with the results for normal-hearing adults (Moore et al. 2000) and normal-hearing teenagers (Moore et al. 2003). This suggests that there is not an age-related effect on masked thresholds in children compared with adults. However, there is a difference between normal-hearing and hearingimpaired children. This may be due to the history of reduced access to the sounds and the limited listening experience in hearing-impaired individuals.

\section{CONCLUSIONS}

The TEN $\mathrm{HL}_{\mathrm{HL}}$ test and the fast-PTC technique can be successfully used with children as young as 8 yr. There is good agreement between outcomes of $\mathrm{TEN}_{\mathrm{HL}}$ test and fast-PTCs, suggesting that the adult criteria for the $\mathrm{TEN}_{\mathrm{HL}}$ test are appropriate in this age group. However, $\mathrm{TEN}_{\mathrm{HL}}$ test results should be interpreted with caution when masked thresholds are 10 to $14 \mathrm{~dB}$ above the TEN level/ERB $\mathrm{E}_{\mathrm{N}}$. Measuring fast-PTCs to reduce false-positive results in these cases is recommended.

\section{ACKNOWLEDGMENTS}

The authors are grateful to children, families, and staff from: Queens Medical Centre in Nottingham, Manchester Inclusion Service for Children and Young People with Hearing Needs or Deafness, and Mary Hare Grammar School. The authors also thank Colette McKay, Karolina Kluk, Chris Plack, and two anonymous reviewers who provided helpful comments on an earlier version of the manuscript.

The study was supported by the National Deaf Children Society and Phonak AG.

Address for correspondence: Alicja N. Malicka, School of Psychological Sciences, University of Manchester, Oxford Road, Manchester M13 9PL, United Kingdom. E-mail: alicja.malicka@manchester.ac.uk.

Received July 23, 2009; accepted September 25, 2009.

\section{REFERENCES}

Allen, P., \& Wightman, F. (1992). Spectral pattern discrimination by children. J Speech Hear Res, 35, 222-233.

Allen, P., \& Wightman, F. (1994). Psychometric functions for children's detection of tones in noise. J Speech Hear Res, 37, 205-215.
Allen, P., \& Wightman, F. (1995). Effects of signal and masker uncertainty on children's detection. $J$ Speech Hear Res, 38, 503-511.

Allen, P., Wightman, F., Kistler, D., et al. (1989). Frequency resolution in children. J Speech Hear Res, 32, 317-322.

Baer, T., Moore, B. C. J., Kluk, K., et al. (2002). Effects of lowpass filtering on the intelligibility of speech in noise for people with and without dead regions at high frequencies. J Acoust Soc Am, 112, $1133-1144$.

Baker, R. J., \& Rosen, S. (2006). Auditory filter nonlinearity across frequency using simultaneous notched-noise masking. J Acoust Soc Am, $119,454-462$.

Buss, E., Hall, J. W., Grose, J. H., et al. (1999). Development of adult-like performance in backward, simultaneous, and forward masking. J Speech Hear Res, 42, 844-849.

British Society of Audiology. (2004). Recommended procedure for pure tone air and bone conduction threshold audiometry with and without masking and determination of uncomfortable loudness levels. United Kingdom: British Society of Audiology.

Cairns, S., Frith, R., Munro, K. J., et al. (2007). Repeatability of the $\mathrm{TEN}(\mathrm{HL})$ test for detecting cochlear dead regions. Int $J$ Audiol, 46, 575-584

Chistovich, L. A. (1957). Frequency characteristics of masking effect. Biofizika, 2, 743-755.

Florentine, M., Buus, S., Scharf, B., et al. (1980). Frequency selectivity in normally-hearing and hearing-impaired observers. J Speech Hear Res, $23,643-669$.

Florentine, M., \& Houtsma, A. J. M. (1983). Tuning curves and pitch matches in a listener with a unilateral, low-frequency hearing loss. $J$ Acoust Soc Am, 73, 961-965.

Glasberg, B. R., \& Moore, B. C. (1990). Derivation of auditory filter shapes from notched-noise data. Hear Res, 47, 103-138.

Hall, J. W., \& Grose, J. H. (1990). Comodulation masking release and auditory grouping. $J$ Acoust Soc Am, 88, 119-125.

Hall, J. W., \& Grose, J. H. (1991). Notched-noise measures of frequency selectivity in adults and children using fixed-masker-level and fixedsignal-level presentation. J Speech Hear Res, 34, 651-660.

Huss, M., \& Moore, B. C. (2005a). Dead regions and pitch perception. $J$ Acoust Soc Am, 117, 3841-3852.

Huss, M., \& Moore, B. C. J. (2003). Tone decay for hearing-impaired listeners with and without dead regions in the cochlea. $J$ Acoust Soc Am, $114,3283-3294$.

Huss, M., \& Moore, B. C. J. (2005b). Dead regions and noisiness of pure tones. Int J Audiol, 44, 599-611.

Irwin, R. J., Stillman, J. A., Schade, A., et al. (1986). The width of the auditory filter in children. J Exp Child Psychol, 41, 429-442.

Kluk, K., \& Moore, B. C. J. (2005). Factors affecting psychophysical tuning curves for hearing-impaired subjects with high-frequency dead regions. Hear Res, 200, 115-131.

Kluk, K., \& Moore, B. C. J. (2006). Detecting dead regions using psychophysical tuning curves: A comparison of simultaneous and forward masking. Int J Audiol, 45, 463-476.

Mackersie, C. L., Crocker, T. L., Davis, R. A., et al. (2004). Limiting high-frequency hearing aid gain in listeners with and without suspected cochlear dead regions. J Am Acad Audiol, 15, 498-507.

Malicka, A. N., Munro, K. J., Baker, R. J., et al. (2009). Fast method for psychophysical tuning curve measurement in school-age children. Int $J$ Audiol, 48, 546-553.

McDermott, H. J., Lech, M., Kornblum, M. S., et al. (1998). Loudness perception and frequency discrimination in subjects with steeply sloping hearing loss: Possible correlates of neural plasticity. J Acoust Soc Am, 104, 2314-2325.

Moore, B. C. J. (1998). Cochlear Hearing Loss. London: Whurr.

Moore, B. C. J. (2001). Dead regions in the cochlea: Diagnosis, perceptual consequences, and implications for the fitting of hearing aids. Trends Amplif, 5, 1-34.

Moore, B. C. J. (2004). Dead regions in the cochlea: Conceptual foundations, diagnosis and clinical applications. Ear Hear, 25, 98-116.

Moore, B. C. J., \& Carlyon, R. P. (2005). Perception of pitch by people with cochlear hearing loss and by cochlear implant users. In C. J. Plack, A. J. Oxenham, R. R. Fay, et al. (Eds). Pitch Perception (pp, 234-277). New York: Springer.

Moore, B. C. J., Glasberg, B. R., Stone, M. A., et al. (2004). New version of the TEN test with calibrations in dB HL. Ear Hear, 25, 478-487. 
Moore, B. C. J., Huss, M., Vickers, D. A., et al. (2000). A test for the diagnosis of dead regions in the cochlea. Br J Audiol, 34, 205-224.

Moore, B. C. J., Killen, T., Munro, K. J., et al. (2003). Application of the TEN test to hearing-impaired teenagers with severe to profound hearing loss. Int $J$ Audiol, 42, 465-474.

Moore, B. C. J., \& Oxenham, A. J. (1998). Psychoacoustic consequences of compression in the peripheral auditory system. Psychol Rev, 105, $108-124$.

Moore, B. C. J., \& Vinay, S. N. (2009). Enhanced discrimination of low-frequency sounds for subjects with high-frequency dead regions. Brain, 132, 524-536.

Murray, N., \& Byrne, D. (1986). Performance of hearing-impaired and normal hearing listeners with various high-frequency cut-offs in hearing aids. Aust J Audiol, 8, 21-28.

Preminger, J. E., Carpenter, R., Ziegler, C. H., et al. (2005). A clinical perspective on cochlear dead regions: Intelligibility of speech and subjective hearing aid benefit. J Am Acad Audiol, 16, 600-613.

Rosen, S., \& Baker, R. J. (1994). Characterising auditory filter nonlinearity. Hear Res, 73, 231-243.

Ruggero, M. A., Rich, N. C., Recio, A., et al. (1997). Basilar-membrane responses to tones at the base of the chinchilla cochlea. J Acoust Soc Am, $101,2151-2163$

Schneider, B. A., Trehub, S. E., Morrongiello, B. A., et al. (1989). Developmental changes in masked thresholds. J Acoust Soc Am, 86, 1733-1742.

Sek, A., Alcantara, J., Moore, B. C., et al. (2005). Development of a fast method for determining psychophysical tuning curves. Int $J$ Audiol, 44, $408-420$.

Small, A. M. (1959). Pure-tone masking. J Acoust Soc Am, 31, 1619-1625.

Stelmachowicz, P. G., Lewis, D. E., Choi, S., et al. (2007). Effect of stimulus bandwidth on auditory skills in normal-hearing and hearingimpaired children. Ear Hear, 28, 483-494.

Stelmachowicz, P. G., Pittman, A. L., Hoover, B. M., et al. (2001). Effect of stimulus bandwidth on the perception of $/ \mathrm{s} /$ in normal- and hearingimpaired children and adults. J Acoust Soc Am, 110, 2183-2190.
Stelmachowicz, P. G., Pittman, A. L., Hoover, B. M., et al. (2002). Aided perception of $/ \mathrm{s} /$ and $/ \mathrm{z} /$ by hearing-impaired children. Ear Hear, 23, $316-324$

Summers, V., Molis, M. R., Musch, H., et al. (2003). Identifying dead regions in the cochlea: Psychophysical tuning curves and tone detection in threshold-equalizing noise. Ear Hear, 24, 133-142.

Veloso, K., Hall, J. W., Grose, J. H. (1990). Frequency selectivity and comodulation masking release in adults and in six-year old children. J Speech Hear Res, 33, 96-102.

Vestergaard, M. (2003). Dead regions in the cochlea: Implications for speech recognition and applicability of articulation index theory. Int $J$ Audiol, 42, 249-261.

Vickers, D. A., Moore, B. C., Baer, T., et al. (2001). Effects of low-pass filtering on the intelligibility of speech in quiet for people with and without dead regions at high frequencies. J Acoust Soc Am, 110, $1164-1175$.

Vinay, Baer, T., Moore, B. C., et al. (2008). Speech recognition in noise as a function of highpass-filter cutoff frequency for people with and without low-frequency cochlear dead regions. J Acoust Soc Am, 123, 606-609.

Vinay, \& Moore, B. C. (2007a). Prevalence of dead regions in subjects with sensorineural hearing loss. Ear Hear, 28, 231-241.

Vinay, \& Moore, B. C. (2007b). Speech recognition as a function of high-pass filter cutoff frequency for people with and without low-frequency cochlear dead regions. J Acoust Soc Am, 122, 542553.

Vinay, \& Moore, B. C. (2007c). Ten(HL)-test results and psychophysical tuning curves for subjects with auditory neuropathy. Int $J$ Audiol, 46, $39-46$.

Werner, L. A., \& Marean, G. C. (1996). Human Auditory Development. Boulder, CO: Westview Press.

Zwicker, E. (1974). On the psychophysical equivalent of tuning curves. In E. Zwicker, \& E. Terhardt (Eds). Facts and Models in Hearing (pp, 132-140). Berlin: Springer-Verlag.

Zwicker, E., \& Schorn, K. (1978). Psychoacoustical tuning curves in audiology. Audiology, 17, 120-140. 